\title{
X-ray photoemission spectroscopic investigation of surface treatments, metal deposition, and electron accumulation on $\ln N$
}

\author{
K. A. Rickert and A. B. Ellis \\ Department of Chemistry, University of Wisconsin-Madison, Madison, Wisconsin 53706 \\ F. J. Himpsel \\ Department of Physics, University of Wisconsin-Madison, Madison, Wisconsin 53706 \\ H. Lu and W. Schaff \\ Department of Electrical and Computer Engineering, Cornell University, Ithaca, New York 14853 \\ J. M. Redwing \\ Department of Materials Science, Pennsylvania State University, University Park, Pennsylvania 16802 \\ F. Dwikusuma and T. F. Kuech ${ }^{\text {a) }}$ \\ Department of Chemical Engineering, University of Wisconsin-Madison, Madison, Wisconsin 53706
}

(Received 17 December 2002; accepted 13 March 2003)

\begin{abstract}
The effects of surface chemical treatments and metal deposition on the InN surface are studied via synchrotron-based photoemission spectroscopy. Changes in the In $4 d$ core level as well as the valence band spectra are reported. The surface Fermi level position, $E_{F}$, relative to the valence band maximum was determined for both $\mathrm{Au}$ and Ti Schottky barriers. $E_{F}$ lies at an energy of $0.7 \mathrm{eV}$ above the valence band maximum for $\mathrm{Au}$ deposited on annealed $\mathrm{InN}$ and $1.2 \mathrm{eV}$ above the valence band maximum for Ti deposited on Ar-sputtered InN. These results that the surface Fermi level lays at or above the conduction band maximum when a value of InN band gap of $0.7-0.9 \mathrm{eV}$ is assumed.
\end{abstract}

(c) 2003 American Institute of Physics. [DOI: 10.1063/1.1573351]

InN is currently being investigated for use in optoelectronic and electronic devices such as high-electron-mobility transistors (HEMTs). ${ }^{1,2}$ The surface electronic properties and even the InN band gap is at present uncertain. Since $\mathrm{InN}$ materials are difficult to prepare, few studies have been published on the nature of the surface properties of InN. Schaff and co-workers have noted that $\mathrm{Hg}, \mathrm{Ti}, \mathrm{Al}$, or $\mathrm{Ni}$ contacts to an $\mathrm{InN}$ surface are ohmic and show low resistance in $I-V$ measurements. ${ }^{1,3} \mathrm{InN}$ may possess a surface Fermi level at or above the conduction band edge leading, potentially, to an electron accumulation layer at the surface, depending on the assumed value of $\mathrm{InN}$ band gap.

Both InAs and $\mathrm{InSb}^{4}$ exhibit an electron accumulation layer near the surface. A narrow conduction band minimum around the center of the Brillouin zone is associated with this charge accumulation layer observed in InAs samples. ${ }^{5}$ The surface electronic properties, in any case, are often dominated by the presence of surface states, which determine the surface Fermi level, $E_{F}$, position. Surface states can pin the Fermi level at an "effective midgap energy" that is sometimes represented by $E_{\mathrm{BP}}$, the branch point energy. ${ }^{6}$ This energy is defined as the energy position within the band gap where the states change from a valence band-like to a conduction band-like character. The $E_{\mathrm{BP}}$ energy position is above the conduction band minimum (CBM) for InAs. ${ }^{7}$ As a result, the InAs surface Fermi level becomes located above the CBM at the surface of InAs and results in a surface charge accumulation layer. ${ }^{8}$

The electron accumulation layer at the InAs surface has

${ }^{a)}$ Electronic mail: kuech@engr.wisc.edu been observed through techniques that include: electron tunneling, ${ }^{8}$ high-resolution electron energy loss spectroscopy, ${ }^{9,10}$ and angle-resolved photoemission spectroscopy. ${ }^{7}$ Surface electron accumulation can be induced by a variety of factors and surface preparations, including surface cleavage, surface reconstruction, and surface adsorption of a few metal atoms. Submonolayer depositions of alkali and transition metals can also lead to an electron accumulation layer in InAs. ${ }^{11-13}$

Metal deposition on a clean InN surface allows the energy level position of the Fermi level to be spectroscopically distinguished in photoemission measurements. An electron accumulation layer can be inferred if the $E_{F}$ lies higher in energy than the conduction band minimum. The photoemission technique required for this analysis has been previously used for a variety of metals on GaN. ${ }^{14,15}$

InN samples were grown via migration-enhanced epitaxy during molecular beam epitaxy. ${ }^{16}$ The sheet carrier concentration was between $9.30 \times 10^{13}$ and $1.95 \times 10^{15} \mathrm{~cm}^{-2}$ and the thickness was between 0.1 and $1.0 \mu \mathrm{m}$ for a given sample. The acquisition of the photoemission spectra, the in situ cleaning techniques and eventual metal deposition were carried out at the Synchrotron Radiation Center in Stoughton, WI. All spectra were collected with an incoming photon energy of $75 \mathrm{eV}$. Untreated samples were loaded and analyzed prior to in situ cleaning.

After initial analysis, samples were subjected to two types of cleaning treatments. The annealed $\mathrm{InN}$ was heated via electron bombardment from a filament behind the sample holder in the analysis chamber. Other InN samples were subjected to Ar ion sputtering at an Ar pressure in the chamber of $4 \times 10^{-5}$ Torr and with an ion-sample current of $6 \mu \mathrm{A}$ for 


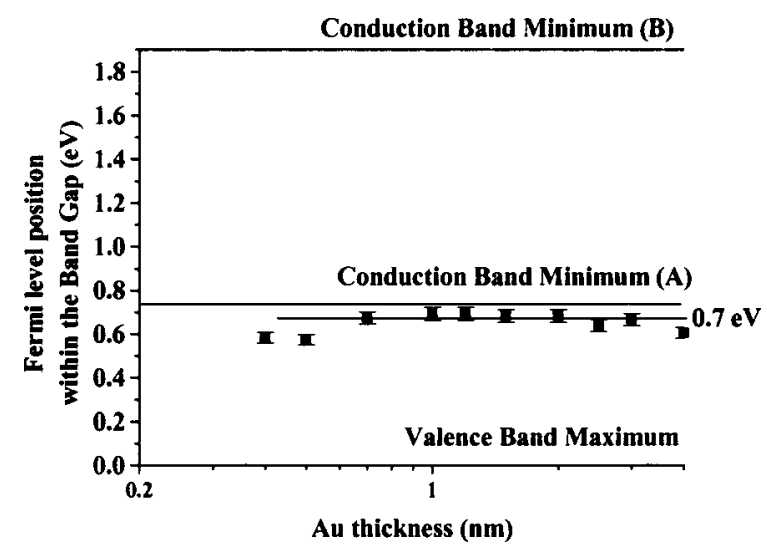

FIG. 1. The $E_{F}$ position with respect to the valence band maximum is shown for the annealed sample as a function of Au coverage, as determined through photoemission spectroscopy. The conduction band minimum (a) is determined with an InN band gap of $0.74 \mathrm{eV}$, while the conduction band minimum (b) is determined with an InN band gap of $1.89 \mathrm{eV}$.

$10 \mathrm{~min}$. The sputtering was done normal to or at a glancing angle of $60^{\circ}$ to the sample surface. After surface cleaning treatments, samples were again analyzed via photoemission spectroscopy before being transferred to the adjacent evaporation chamber for metal deposition.

$\mathrm{Au}$ or Ti was deposited on the samples in sub-nm quantities at a rate of $0.01 \mathrm{~nm} / \mathrm{s}$. After each deposition, the $\mathrm{In} 4 d$ core level and Fermi edge photoemission spectra were collected. This metal deposition process continued until the total metal thickness was $15-20 \mathrm{~nm}$. The first derivatives of the Fermi edge spectra were fit to a Gaussian function to accurately determine the Fermi level energy from each spectrum. In order to determine the Fermi level position relative to the band edges, the In $4 d$ core level to valence band maximum energy difference $\left(E_{V-C}\right)$ was first determined on the sample prior to metal deposition. The In $4 d$ core level and metal Fermi edge were then measured at each coverage of metal overlayer and used to determine the In $4 d$ binding energy $\left(E_{B}\right)$. Once these values are known, the $E_{F}$ position within the gap with respect to the valence band can be determined through Eq. (1):

$$
E_{F}-\mathrm{VBM}=E_{B}-E_{V-C}
$$

and the $n$-InN Schottky barrier height, $\Phi_{B, n}$, through

$$
\Phi_{B, n}=E_{\text {gap }}-E_{B} .
$$

The determination of the barrier height is therefore dependent on an accurate value of the band gap, $E_{\text {gap }}$.

The In $4 d$ core level and valence band region spectra for an annealed InN sample were compared. The thermal annealing shifts the In $4 d$ core level by $\sim 0.5 \mathrm{eV}$ to higher kinetic energy, and the spin-orbit-split $4 d_{5 / 2}$ and $4 d_{3 / 2}$ peaks were not observed. There is also measurable change in the valence band spectrum. A shoulder feature, seen on the higher kinetic energy side, becomes more prominent postannealing. The edge of this feature can be fit to a linear function and the zero-photoemission intensity intercept can be used as the valence band maximum (VBM). This VBM was used along with a Gaussian fit of the In $4 d$ core level on the annealed sample to calculate an $E_{V-C}$ value of $16.9 \mathrm{eV}$. After Au was deposited on the surface, the binding energy of the In $4 d$ Downloaded 06 Mar 2007 to 128.104 .198 .190 . Redistribution subje


FIG. 2. Photoemission spectra for an InN sample, first untreated, then $\mathrm{Ar}$ sputtered at a glancing angle, was and then sputtered twice at normal incidence. The four spectra were collected with a photon energy of $75 \mathrm{eV}$. (a) The In $4 d$ core level spectrum shows the appearance of the $4 d_{5 / 2}$ and $4 d_{3 / 2}$ peaks after the completion of the first normal incidence sputter treatment. (b) In the valence band region, the two new features are indicated by I and II, in addition to the VBM feature, and the Fermi edge, $E_{F}$, which appear after the first normal incidence sputter treatment.

core level was, again, determined and the Fermi edge was determined as described earlier. A plot of the Fermi level position within the band gap of annealed InN with Au coverage is shown in Fig. 1. The $E_{F}$ position remains at a value of $0.7 \pm 0.1 \mathrm{eV}$ above the valence band maximum. The band gap of InN is a matter of controversy at present. The two conduction band minimum lines on Fig. 1 indicate the two approximate values of the $\mathrm{InN}$ band gap presently discussed in the literature. An InN band gap of $1.89 \mathrm{eV}$, was determined through optical absorption techniques. ${ }^{17-19}$ Recent photoluminescence studies indicate the band gap of InN may be as small as $0.7-0.8 \mathrm{eV}^{20-22}$ Depending on the value chosen for the band gap in Eq. (2), the surface barrier height of $\mathrm{Au}$ on annealed InN could either be considered to be essentially zero or $1.2 \pm 0.1 \mathrm{eV}$. The choice of band gap also affects the conclusions drawn about the presence of an electron accumulation layer in InN. The use of the smaller band gap would indicate the presence of an electron accumulation layer, while the $1.89 \mathrm{eV}$ band gap would lead to an absence of an electron accumulation layer with our specific samples and preparation techniques.

The photoemission spectra for an Ar-sputtered $\mathrm{InN}$ sample are shown in Fig. 2. The sample was first sputtered at an angle of $60^{\circ}$ before being sputtered at normal incidence to the sample surface. Normal incidence sputtering leads to the appearance of two peaks in the In $4 d$ core level spectrum to AlP license or copyright, see http://apl.aip.org/apl/copyright.jsp 




FIG. 3. The $E_{F}$ position with respect to the valence band maximum is shown for the Ar-sputtered sample as a function of Ti coverage, as determined through photoemission spectroscopy. The conduction band minimum (a) is determined with an $\mathrm{InN}$ band gap of $0.74 \mathrm{eV}$ while the conduction band minimum (b) is determined with an InN band gap of $1.89 \mathrm{eV}$.

[Fig. 2(a)] with an energy difference corresponding to the expected spin-orbit splitting of $0.86 \mathrm{eV}$ for In $4 d_{5 / 2}$ and In $4 d_{3 / 2}$ which are expected to originate from islands of metallic In on the surface. ${ }^{23}$ A contribution from the original InN peak, is visible in the spectrum, even after three sputtering cycles. Additional changes can be observed in the valence band region, Fig. 2(b). The initial valence band spectrum for the untreated InN sample is consistent with experimental results reported in the literature. ${ }^{24}$ There are two major features, labeled I and II in Fig. 2(b), with the same height ratio and width described previously by Guo et al. ${ }^{24}$ After the sample was sputtered repeatedly, feature II splits into two peaks. This peak splitting is consistent with the calculated InN density of states. ${ }^{25} \mathrm{~A}$ Fermi edge at higher kinetic energy than the VBM becomes visible after the normal incidence sputtering, due to the formation of metallic In islands on the surface. ${ }^{26}$ The valence band edge also becomes more pronounced upon sputtering. The In $4 d$ core level from the untreated sample is used along with a linear fit of the valence band edge, also from the untreated sample, to determine the value of $E_{V-C}$. The $E_{V-C}$ for this sputtered $\mathrm{InN}$ is again 16.9 $\mathrm{eV}$, consistent with what was reported for the annealed $\mathrm{InN}$ sample. The In $4 d$ core level spectra are fit to three Gaussian functions in order to determine the binding energy for the In $4 d$ core level associated with InN. The Fermi level position within the band gap of sputtered $\mathrm{InN}$ determined with the $E_{V-C}$ and the $E_{B}$ values is shown in Fig. 3. The large error bars are due to the difficulty associated with peak-fitting the broader, lower kinetic energy In $4 d$ peak in the presence of the two higher intensity metallic In $4 d$ core levels. This measured $E_{F}$ position is $1.2 \pm 0.1 \mathrm{eV}$ above the valence band maximum. Again, if the InN band gap is $1.89 \mathrm{eV}$, then $E_{F}$ would lead to a barrier height of $0.7 \pm 0.1 \mathrm{eV}$. Alternatively, if the band gap value of $\sim 0.7 \mathrm{eV}$ is employed, then $E_{F}$ would be at or in the conduction band. This Fermi level position would be consistent with the observed ease of ohmic contact formation ${ }^{1}$ and would be consistent with the presence of an electron accumulation layer.

$\mathrm{Au}$ deposited on annealed $\mathrm{InN}$ yields a Fermi level 0.7 $\mathrm{eV}$ above the valence band maximum, while Ti deposited on sputtered InN yields a Fermi level $1.2 \mathrm{eV}$ above the valence band maximum. If the smaller value of the band gap for $\mathrm{InN}$, $0.7 \mathrm{eV}$, is assumed, then neither metal forms a Schottky barrier on InN and an electron accumulation layer would be possible.

This work was supported by the National Science Foundation. Research and facility support at the Synchrotron Radiation Center at the UW-Madison (DMR-0084402) is gratefully acknowledged.

${ }^{1}$ W. J. Schaff, H. Lu, J. Hwang, and H. Wu, in Growth of InN for Heterojunction Field Effect Transistor Applications by Plasma Enhanced MBE, Cornell University (IEEE, New York, 2000) pp. 225-231.

${ }^{2}$ S. Nakamura, T. Mukai, and M. Senoh, Appl. Phys. Lett. 64, 1687 (1994).

${ }^{3}$ J. Wu, W. Walukiewicz, K. M. Yu, J. W. Ager, E. E. Haller, H. Lu, W. J. Schaff, Y. Saito, and Y. Nanishi, Appl. Phys. Lett. 80, 3967 (2002).

${ }^{4}$ V. Y. Aristov, V. M. Zhilin, C. Grupp, A. Taleb-Ibrahimi, H. J. Kim, P. S. Mangat, P. Soukiassian, and G. Le Lay, Appl. Phys. Lett. 166, 263 (2000).

${ }^{5}$ M. C. Håkansson, L. S. O. Johansson, C. B. M. Andersson, U. O. Karlsson, L. Ö. Olsson, J. Kanski, L. Ilver, and P. O. Nilsson, Surf. Sci. 374, 73 (1997).

${ }^{6}$ J. Tersoff, Phys. Rev. Lett. 52, 465 (1984).

${ }^{7}$ L. Ö. Olsson, C. B. M. Andersson, M. C. Håkansson, J. Kanski, L. Ilver, and U. O. Karlsson, Phys. Rev. Lett. 76, 3626 (1996).

${ }^{8}$ D. C. Tsui, Phys. Rev. Lett. 24, 303 (1970).

${ }^{9}$ M. Noguchi, K. Hirakawa, and T. Ikoma, Phys. Rev. Lett. 66, 2243 (1991).

${ }^{10}$ T. D. Veal and C. F. McConville, Phys. Rev. B 64, 085311-1 (2001).

${ }^{11}$ M. G. Betti, G. Bertoni, V. Corradini, V. De Renzi, and C. Mariani, Surf. Sci. 454-456, 539 (2000).

${ }^{12}$ V. Y. Aristov, G. Le Lay, P. Soukiassian, K. Hricovini, J. E. Bonnet, J. Osvald, and O. Olsson, Europhys. Lett. 26, 359 (1994).

${ }^{13}$ M. Morgenstern, M. Getzlaff, D. Haude, R. Wiesendanger, and R. L. Johnson, Phys. Rev. B 61, 13805 (2000).

${ }^{14}$ K. A. Rickert, J. K. Kim, J.-L. Lee, A. B. Ellis, F. J. Himpsel, F. Dwikusuma, and T. F. Kuech, J. Appl. Phys. 92, 1 (2002).

${ }^{15}$ K. A. Rickert, J. K. Kim, J.-L. Lee, F. J. Himpsel, A. B. Ellis, and T. F. Kuech, Mater. Res. Soc. Symp. Proc. 693, I13.4.1-6 (2002).

${ }^{16} \mathrm{H}$. Lu, W. J. Schaff, J. Hwang, H. Wu, W. Yeo, A. Pharkya, and L. F. Eastman, Appl. Phys. Lett. 77, 2548 (2000).

${ }^{17}$ T. L. Tansley and C. P. Foley, Electron. Lett. 20, 1066 (1984).

${ }^{18}$ T. L. Tansley and C. P. Foley, J. Appl. Phys. 59, 3241 (1986).

${ }^{19}$ V. Y. Davydov, A. A. Klochikhin, R. P. Seisyan, V. V. Emtsev, S. V. Ivanov, F. Bechstedt, J. Furthmuller, H. Harima, A. V. Mudryi, J. Aderhold, O. Semchinova, and J. Graul, Phys. Status Solidi B 229, R1 (2002).

${ }^{20}$ V. Y. Davydov, A. A. Klochikhin, V. V. Emtsev, S. V. Ivanov, V. V. Vekshin, F. Bechstedt, J. Furthmuller, H. Harima, A. V. Mudryi, A. Hashimoto, A. Yamamoto, J. Aderhold, J. Graul, and E. E. Haller, Phys. Status Solidi B 230, R4 (2002).

${ }^{21}$ J. Wu, W. Walukiewicz, K. M. Yu, J. W. I. Ager, E. E. Haller, H. Lu, and W. J. Schaff, Appl. Phys. Lett. 80, 4741 (2002).

${ }^{22}$ J. Wu, W. Walukiewicz, K. M. Yu, J. W. I. Ager, E. E. Haller, H. Lu, W. J. Schaff, Y. Saito, and Y. Nanishi, Appl. Phys. Lett. 80, 3967 (2002).

${ }^{23}$ D. M. Poirier and J. H. Weaver, Surf. Sci. Spectra 2, 224 (1994).

${ }^{24}$ Q. X. Guo, M. Nishio, H. Ogawa, A. Wakahara, and A. Yoshida, Phys. Rev. B 58, 15304 (1998).

${ }^{25}$ C. P. Foley and T. L. Tansley, Phys. Rev. B 33, 1430 (1986).

${ }^{26}$ P. De Padova, C. Quaresima, P. Perfetti, R. Larciprete, R. Brochier, C. Richter, V. Ilakovac, P. Bencok, C. Teodorescu, V. Y. Aristov, R. L. Johnson, and K. Hricovini, Surf. Sci. 482-485, 587 (2001). 